\title{
ARTICLE
}

\section{Rho/Rock signal transduction pathway is required for MSC tenogenic differentiation}

\author{
Edward Maharam ${ }^{1}$, Miguel Yaport ${ }^{1}$, Nathaniel L Villanueva ${ }^{1}$, Takintope Akinyibi ${ }^{1}$, Damien Laudier ${ }^{1}$, Zhiyong He ${ }^{2,3}$, \\ Daniel J Leong ${ }^{2,3}$ and Hui B Sun ${ }^{2,3}$
}

Mesenchymal stem cell (MSC)-based treatments have shown promise for improving tendon healing and repair. MSCs have the potential to differentiate into multiple lineages in response to select chemical and physical stimuli, including into tenocytes. Cell elongation and cytoskeletal tension have been shown to be instrumental to the process of MSC differentiation. Previous studies have shown that inhibition of stress fiber formation leads MSCs to default toward an adipogenic lineage, which suggests that stress fibers are required for MSCs to sense the environmental factors that can induce differentiation into tenocytes. As the Rho/ROCK signal transduction pathway plays a critical role in both stress fiber formation and in cell sensation, we examined whether the activation of this pathway was required when inducing MSC tendon differentiation using rope-like silk scaffolds. To accomplish this, we employed a loss-of-function approach by knocking out ROCK, actin and myosin (two other components of the pathway) using the specific inhibitors Y-27632, Latrunculin A and blebbistatin, respectively. We demonstrated that independently disrupting the cytoskeleton and the Rho/ ROCK pathway abolished the expression of tendon differentiation markers and led to a loss of spindle morphology. Together, these studies suggest that the tension that is generated by MSC elongation is essential for MSC teno-differentiation and that the Rho/ROCK pathway is a critical mediator of tendon differentiation on rope-like silk scaffolds.

Bone Research (2015) 3, 15015; doi:10.1038/boneres.2015.15; Published online: 6 October 2015

\section{INTRODUCTION}

Tendon injuries are a common clinical problem. ${ }^{1-3}$ Following injury, tendons exhibit an ineffective repair response that is often characterized by scar formation. ${ }^{4}$ Severe tendon injuries often require surgical intervention, but the structure and function of repaired tendons are inferior when compared to non-injured tendons. ${ }^{5-6}$ Consequently, there is a clear need for new strategies that can fully restore tendon function following injury.

Advances in tissue engineering have shown promise for the treatment of tendon injuries using mesenchymal stem cells (MSCs) and scaffolds. ${ }^{6}$ MSCs can be isolated from various tissues, including bone marrow, adipose tissue, and tendons, ${ }^{7-9}$ and they have the ability to differentiate into tissues of musculoskeletal lineage, including tenocytes. ${ }^{10}$ Bombyx mori (silkworm) silk is a promising material for use as a scaffold because it has strong mechanical properties that slowly degrade over time, allowing for newly formed tissue to gradually bear the mechanical load of healing tissue." "Silk scaffolds also support cell implantation. The expression levels of tendon matrix and teno-phenotypic associated proteins, such as collagen I, collagen III, and tenascin-C, were shown to be upregulated when MSCs were seeded onto aligned silk fibers, suggesting an enhancement of MSC tenogenesis. ${ }^{12-13}$ In vivo, MSC-seeded silk scaffolds were able to foster the development of a regenerated ligament, which exhibited increased expression levels of collagen I, collagen III, and tenascin- $C$ and maintained a maximum tensile load 24 weeks after implantation. ${ }^{14}$

The above discussed studies, which were performed in the absence of tenogenic inductive media, suggest that cues from the matrix microenvironment may promote MSC teno-commitment and differentiation. ${ }^{15}$ However, the underlying mechanism behind this phenomenon is largely unknown. Previous work has elucidated the

'Leni and Peter W. May Department of Orthopedics, Icahn School of Medicine at Mount Sinai, New York, NY, USA ${ }^{2}$ Departments of Orthopedic Surgery, Albert Einstein College of Medicine, Bronx, NY, USA ${ }^{3}$ Radiation Oncology, Albert Einstein College of Medicine, Bronx, NY, USA Correspondence: Hui B Sui (Herb.sun@einstein.yu.edu)

Received: 2 December 2014; Revised: 20 March 2015; Accepted: 20 April 2015 
importance of cell tension, specifically via actin and nonmuscle myosin interactions, in regulating cell differentiation and commitment. ${ }^{16}$ After treating MSCs with either cytochalasin D, an actin inhibitor, or with blebbistatin, a non-muscle myosin II inhibitor, they either remained largely undifferentiated or defaulted to an adipogenic fate. ${ }^{16}$

The maintenance of cytoskeletal tension and cell shape is partially governed by the Rho family of GTPases. ${ }^{16-19}$ The formation of adherens junctions and focal adhesions, both of which are mediated in part by the action of Rho family GTPases, are important in the cell-cell interactions that are responsible for cell commitment. ${ }^{17,20}$ Furthermore, the Rho/ROCK signaling pathway, one of the most wellstudied Rho family GTPase-effector pathways, is the major regulator of stress fiber formation under most physiological conditions. ${ }^{21}$ The Rho/ROCK pathway has been suggested to play a role in the differentiation of MSCs into several connective tissue lineages, such as into bone or adipose tissue, ${ }^{16,20}$ and also in tenogenic differentiation in response to mechanical stretch. ${ }^{22}$

In this study, we examined how the attachment, elongation, and proliferation of MSCs on silk fibers trigger the cell-signaling events that lead to tenogenic-like differentiation. We hypothesized that the attachment of MSCs onto rope-like silk fibers and their associated morphological changes are the determining factors of tenogenic differentiation of MSCs in this context. Furthermore, as the Rho/ ROCK pathway has been shown to be associated both with stress fiber formation and cell sensation, we postulate that it is required for MSC tendon differentiation.

\section{METHODS AND MATERIALS}

\section{Cell culture}

Mouse C3H10T-1/2 MSCs were acquired from ATCC and were maintained using growth media that consisted of Dulbecco's Modified Eagle Medium (DMEM) that was supplemented with $1 \%$ penicillin-streptomycin antibiotics (100 U. $\mathrm{mL}^{-1}$ ) and 10\% fetal bovine serum (FBS; HyClone Logan). The cells were cultured at $37{ }^{\circ} \mathrm{C}$ in a humidified atmosphere of $5 \% \mathrm{CO}_{2}$. The growth media was replaced every $48 \mathrm{~h}$, and when cells reached $80 \%$ confluence they were dissociated using a $0.05 \%$ Trypsin-EDTA solution (Cellgro) and were either subcultured or used for experimentation. MSCs $\left(1.5 \times 10^{6} \mathrm{cells}\right)$ in DMEM were laid onto a scaffold and placed at $37^{\circ} \mathrm{C}$ with $5 \% \mathrm{CO}_{2}$ for $2 \mathrm{~h}$. Following this procedure, $2 \mathrm{~mL}$ DMEM was added to the cells, and they were cultured at $37^{\circ} \mathrm{C}$ with $5 \% \mathrm{CO}_{2}$ with daily replacement of culture media. For time course studies, cells cultured on rope-like silk scaffolds were initially harvested after an initial 2-h attachment period and were designated as the 0-h group. The following additional groups were harvested at the corresponding times after the attachment period: $4 \mathrm{~h}, 12 \mathrm{~h}, 1$ day, and 7 days.
Preparation of custom rope-like silk scaffolds and silkprotein coated culture dishes

Raw Bombyx mori 20/22 density silk fibers were provided by DESCO (Zürich, Switzerland ${ }^{23}$ ). Scaffolds were fabricated by wrapping the silk fibers (without overlapping) around a $20 \mathrm{~cm} \times 20 \mathrm{~cm}$ Plexiglas plate with $2 \mathrm{~mm}$-thick rubber borders until the fibers reached a width of approximately $0.3 \mathrm{~cm}$. The fibers were glued together using Marine Adhesive Sealant Fast Cure 5200 (3M Marine) at fixed intervals, leaving approximately $2 \mathrm{~cm}$ of exposed silk. After allowing them to cure for $24 \mathrm{~h}$, the scaffolds were separated by scoring the adhesive that had previously been placed between the strips of scaffold. The scaffolds were then washed with $70 \%$ ethanol, and sericin proteins were removed via a $24 \mathrm{~h}$ soak in $0.05 \%$ Trypsin-EDTA at $37^{\circ} \mathrm{C}$ and $5 \% \mathrm{CO}_{2} \cdot{ }^{24}$ Prior to seeding, the scaffolds were washed three times in PBS and once in DMEM.

To seed MSCs onto a control silk protein-coated culture surface, raw silk fibers were digested in $0.05 \%$ Trypsin-EDTA for $24 \mathrm{~h}$ at $1 \mathrm{~g}$ of silk with $40 \mathrm{~mL}$ of Trypsin-EDTA. Following this procedure, the fibers were washed five times with deionized water before being dissolved in $\mathrm{CaCl}_{2}-\mathrm{CH}_{3} \mathrm{CH}_{2} \mathrm{OH}$ $\mathrm{H}_{2} \mathrm{O}$ (molar ratio $1: 2: 8$ ) at $78 \pm 2{ }^{\circ} \mathrm{C}$ with continuous stirring for $2 \mathrm{~h}$. The solution was then dialyzed against distilled water using Snake Skin Pleated Dialysis Tubing (Thermo Scientific, MWCO3500); the distilled water was refreshed daily for 5 days. The silk solution was then filtered through a disposable sterilization filter unit (Nalgene). A total of $3 \mathrm{~mL}$ of solution was poured into the bottom of a cell culture dish. After being allowed to sit for $6 \mathrm{~h}$ under a sterile hood, the remaining fluid was vacuumed off, leaving a thin silk film on the bottom of the dish that could be used for cell culture. To perform experiments, equivalent numbers of MSCs $\left(1.5 \times 10^{6}\right.$ cells $)$ were seeded into $35 \mathrm{~mm}$ dishes.

Immunocytochemistry

The scaffolds were removed from the growth media and rinsed several times with PBS. The cells on the scaffolds were then fixed with $1 \%$ formalin for $1 \mathrm{~h}$ at room temperature. After several washes with PBS, the scaffolds were incubated with $0.3 \%$ hydrogen peroxide for 10 min at room temperature, followed by three washes with PBS. To facilitate the detection of scleraxis proteins, membrane permeabilization was achieved by incubating the scaffolds for 10 min in $0.5 \%$ Triton-X that was diluted in PBS. A serum-free protein block (Dako, US) was then added to the scaffolds for $1 \mathrm{~h}$ at room temperature, followed by the addition of primary antibodies in diluent solution (Dako). Anti-Scx (Abcam, 1:300), anti-Tnmd (Santa Cruz, 1:300), and anti-Actin (Santa Cruz, 1:300) primary antibodies were added to individual scaffolds and incubated at room temperature for $1 \mathrm{~h}$. As a negative control, the scaffolds were incubated with titered rabbit serum instead 
of primary antibodies. Scaffolds were then washed at least three times in a solution of PBS that contained $0.1 \%$ Tween-20 (PBS-T); washes lasted for 5 min each. The following fluorochrome-conjugated secondary antibodies were applied: Scx (Alexafluor 568, Molecular Probes, 1:1 000), Tnmd (Alexafluor 488, Molecular Probes, 1:1 000), and $\beta$-actin (Alexafluor 488, 1:1 000). The scaffolds were washed in PBS at least three times and remained submerged in buffered solution for imaging. The samples were imaged using a Leica confocal microscope, and the images were captured with Leica Application Suite Software.

\section{Pharmacological agents}

The following pharmacological agents were employed: $0.5 \mu \mathrm{mol} \cdot \mathrm{L}^{-1}$ Latrunculin A, which is a G-actin polymerization inhibitor (Calbiochem), $10 \mu \mathrm{mol} \cdot \mathrm{L}^{-1} \mathrm{Y}$-27632 dihydrochloride, which is a ROCK inhibitor (Tocris Bioscience), $50 \mu \mathrm{mol} \cdot \mathrm{L}^{-1}$ Blebbistatin (EMD Biosciences), which is a non-muscle myosin $\|$ inhibitor, and $5 \mathrm{mmol} \cdot \mathrm{L}^{-1}$ Cytochalasin D, which is a non-specific actin polymerization inhibitor (Calbiochem). Following the 2-h attachment period, aliquots of DMEM that each contained one of the above listed pharmacological agents in $2 \%$ DMSO were individually applied to the scaffolds. DMEM with $2 \%$ DMSO was used as a control. The scaffolds were cultured at $37^{\circ} \mathrm{C}$ and $5 \% \mathrm{CO}_{2}$ for $24 \mathrm{~h}$ and then harvested to assess mRNA expression.

RNA isolation, CDNA synthesis, and quantitative PCR Total RNA was extracted using an RNeasy mini kit (Qiagen) with DNase treatment. RNA was quantified with a Nanodrop spectrophotometer (Thermo Fisher Scientific, Wilmington, DE) and then was reverse-transcribed (RT) using oligo (dT) primers. A 10 ng sample of total RNA was analyzed by real-time PCR to assess the expression of the following housekeeping genes: scleraxis $(S C X)$; tenomodulin (Tnmd); collagenlal (Colla I); nuclear factor of activated T-cells, cytoplasmic 4 (Nfatc4); nucleostemin (Nst) octamer-binding transcription factor 4 (Oct4); sex-determining region Y-box 9 (Sox9); collagen 2al (Col2al); runt-related transcription factor 2 (Runx2); alkaline phosphatase (Alp); glyceraldehyde 3-phosphate dehydrogenase (Gapdh); hypoxanthine-guanine phosphoribosyltransferase 1 (Hprtl); and phosphoglycerate kinase 1 (Pgkl). The PCR primers pairs were shown in Table 1. Quantitative real-time PCR with SYBR Green was performed. The expression values of GAPDH under each treatment condition were averaged and used as a denominator to determine relative gene expression. Data were analyzed for significance $(P<0.05)$ using one-way ANOVA and Student's t-tests, as appropriate.
Table 1. PCR primers pairs

\begin{tabular}{lll}
\hline Gene & \multicolumn{1}{c}{ Forward } & \multicolumn{1}{c}{ Reverse } \\
\hline Scx & 5'-AAACAGATCTGCACCTTCTG-3' & 5'-TCAGATCAGGTCCAAAGTGG-3' \\
Tnmd & 5'-ATGACATGGAGCACACTTC-3' & 5'-AAGTAGATGCCAGTGTATCC-3' \\
Collal 1 5'-TGACTGGAAGAGCGGAGAGT-3' & 5'-GTTCGGGCTGATGTACCAGT-3' \\
Nfatc4 & 5'-ACCGGATTACTGGCAAGATG-3' & 5'-CTGTCTCACCCTTCCGTAGC-3' \\
Nst & 5'-GTCTGATCTAGTACCAAAGG-3' & 5'-CTTGCCACAGCAGCAGATTTGC-3' \\
Oct4 & 5'-AGTGGGGCGGTTTGAGTAA-3' & 5'-TTCCAAAGAGAACGCCCAGG-3' \\
Sox9 & 5'-TCCCCGCAACAGATCTCCTA-3' & 5'-AGGTGGAGTAGAGCCCTGAG-3' \\
Col2al & 5'-GCCAGGATGCCCGAAAATTAG-3' & 5'-CGCACCCTTTCTCCCTTGT-3' \\
Runx2 & 5'-CGTCGTCAGACCGAGAAGTG-3' & 5'-GGATCTCGGGGCGACAGA-3' \\
Alp & 5'-TGGTCACAGCAGTTGGTAGC-3' & 5'-ACCCTGAGATTCGTCCCTCG-3' \\
Gapdh & 5'-CTGCCAAGTATGATGACATC-3' & 5'-TGTCATACCAGGAAATGAGC-3' \\
Hprt1 & 5'-GAAGTGTTGGATACAGGCCAGA-3' 5'-TTGCGCTCATCTTAGGCTTTG-3' \\
Pgk1 & 5'-GGGAACCAAGTCACTCATGGA-3' & 5'-GGAGCTCTAGACTGGCACCG-3' \\
\hline
\end{tabular}

Western blot analysis of scleraxis expression

Cells were harvested in lysis buffer and then cleared by centrifugation at $13000 \mathrm{rpm} \cdot \mathrm{min}^{-1}$ for $10 \mathrm{~min}$ at $4{ }^{\circ} \mathrm{C}$. A $20 \mu \mathrm{g}$ sample of each lysate was analyzed on a $12 \%$ SDSPAGE gel and then transferred to a polyvinylidene difluoride membrane and blocked with $5 \%$ non-fat milk before being probed with primary rabbit antibody against scleraxis, at a concentration of $2 \mu \mathrm{mol} \cdot \mathrm{L}^{-1}$ (Santa Cruz).

\section{RESULTS}

Silk fibers promoted tenocyte differentiation of MSCs The custom-made silk scaffolds enabled MSCs to attach and elongate along the fibers, remain viable, and differentiate on the fibers without any additional inductive stimulus. Figure la shows an image of a custom-made, parallel-fiber scaffold. At 7-days post-seeding, C3H10T-1/ 2 MSCs were able to attach to the scaffold (Figure 1b), and they aligned along the scaffold fibers (Figure 1c). To determine the tenogenic potential of these MSCs when seeded on silk scaffolds, the relative gene expression of scleraxis $(S C x)$, an early marker of tendon differentiation, ${ }^{25}$ was assayed. Scx expression increased from 4-h to 24-h post-seeding and began to plateav at $24 \mathrm{~h}$; the same level was maintained at 7-days post-seeding (Figure 1d). These data indicate that rope-like silk scaffolds provide a microenvironment that not only facilitates the attachment, elongation, and growth of MSCs but that also may induce the tenocytic differentiation of MSCs without the need of an additional inductive stimulus.

Tenocyte differentiation is induced by the topography of the three-dimensional structure of the silk fibers

To determine whether the tenocyte differentiation that was promoted by our silk scaffolds occurred in response to the topography of the silk fibers or to the actual silk proteins, we analyzed the expression levels of tenogenic-phenotype-related markers and stem cell markers in MSCs that were seeded onto the silk scaffolds versus 

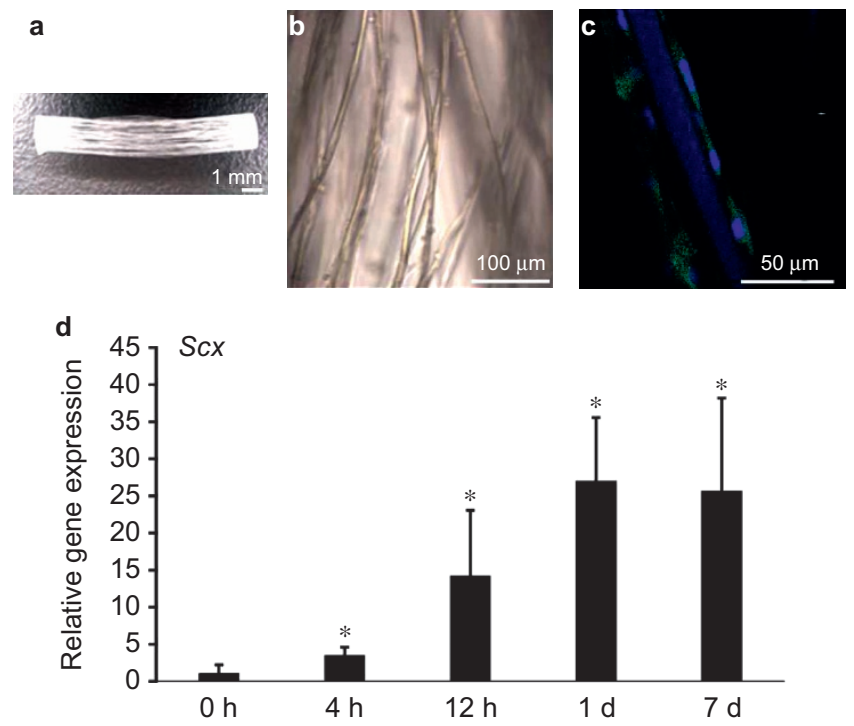

Figure 1. MSC attachment, arrangement, viability, and gene expression after being grown on a scaffold for 7 days. (a) Image of unseeded scaffold. (b) Brightfield image of MSCs attaching to scaffold. (c) Immunocytochemistry image of MSCs grown on a silk scaffold for 7 days (blue $=$ DAPI, green $=\beta$-actin). $(\mathbf{d})$ Relative mRNA expression of $S c x$ at 0,4 , and $12 \mathrm{~h}$, and 1 day and 7 days after seeding MSCs onto the silk scaffold. Data represent three independent experiments per assay. $* P<0.05$ compared with control. those that were grown in silk-coated culture dishes. The MSCs that were cultured on the silk scaffolds exhibited significantly higher expression of SCx and tenomodulin (Tnmd), a late marker of tendon formation, ${ }^{26}$ compared to those that were cultured on silk-coated plates (Figure 2a), indicating that tenogenic differentiation was induced by the physical properties of the silk fibers and not by the chemical components of silk. The MSCs that were seeded on the silk scaffolds also exhibited significantly reduced expression of nucleostemin (Nst) and octamer-binding transcription factor 4 (Oct4), which are markers of undifferentiated $\mathrm{MSCs}^{27}$ when compared to those that were grown on the silk-coated plates (Figure 2a). Although the cell seeding density may have varied between the experiments using silk scaffolds and those using silk-coated plates, these data suggest that MSCs that are cultured on silk-coated plates remain largely undifferentiated, whereas those that are cultured on silk scaffolds differentiate toward a tenogenic lineage. Furthermore, regardless of whether MSCs were seeded on a silk-coated surface or a silk scaffold, the mRNA expression levels of the chondrogenic markers Sox9 and Col2al were respectively unchanged or undetected, and the mRNA expression levels of the osteogenic markers runt-

a

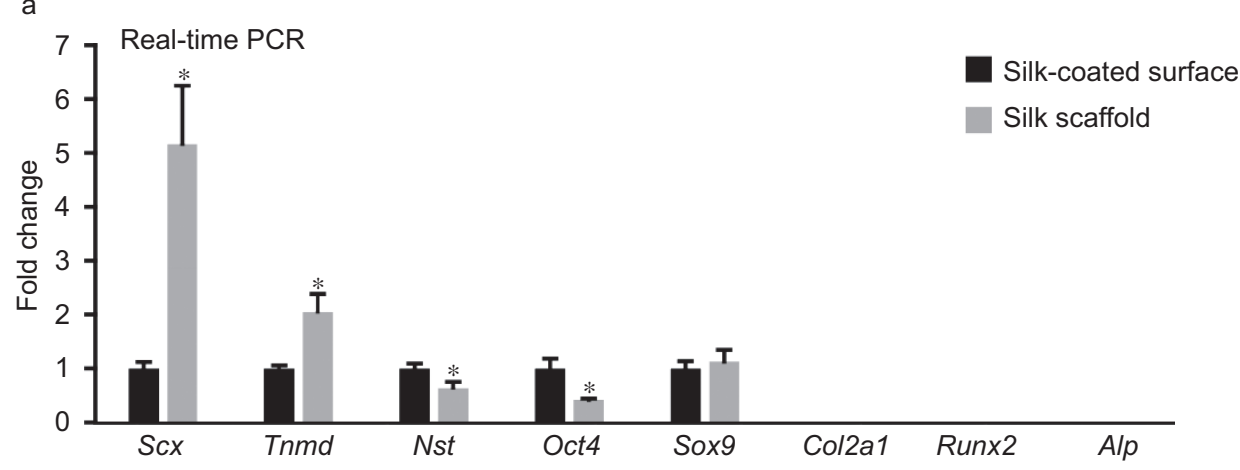

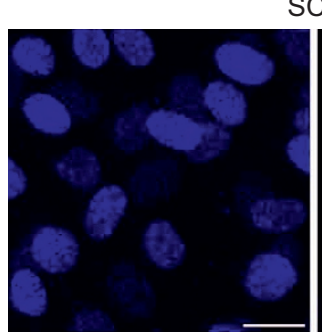

Silk-coated surface
SCX

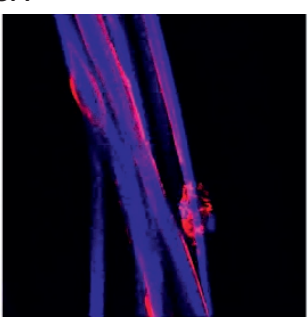

Silk scaffold

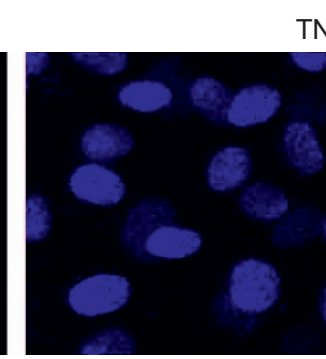

Silk-coated surface
TNMD

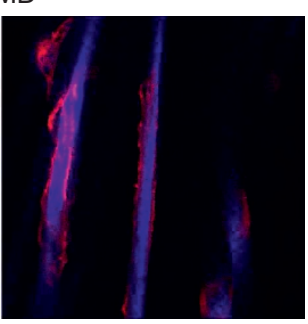

Silk scaffold

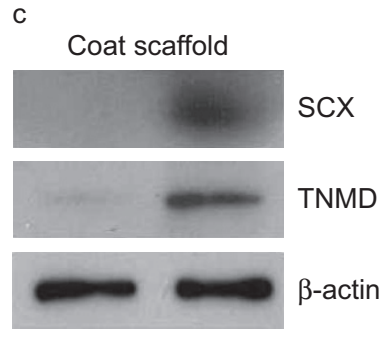
Figure 2. MSC differentiation toward tenocytes on rope-like silk scaffold measured by gene and protein expression. (a) Relative mRNA expression
levels of $S c x$, Tnmd, Nst, Oct4, Sox 9 , Col2a1, Runx2, and Alp in MSCs grown on silk-coated plates or silk scaffolds at 7 days after MSCs were seeded. (b) Immunocytochemistry staining (blue = DAPI, red = SCX or TNMD) and (c) western blot of SCX and TNMD in cells that were seeded on silkcoated plates and silk scaffolds. Data represent three independent experiments per assay. $* P<0.05$ compared to MSCs grown on silk-coated plates. Scale bar $=50 \mu \mathrm{m}$. 
related transcription factor 2 (Runx2) and alkaline phosphatase (A/p) were undetected (Figure 2a). These findings may indicate that MSCs specifically differentiate toward a tenogenic lineage. Immunocytochemistry and western blotting confirmed the lack of SCX and TNMD expression in MSCs cultured on silk protein-coated plates, whereas expression of SCX and TNMD was detected in MSCs grown on silk scaffolds (Figure 2b). These results strongly suggest that the topography (i.e., the elongated physical property) of the silk fibers, and not the protein/chemical components of the silk, is the determining factor of the tenogenic differentiation of MSCs in this context.

Tenogenic morphogenesis is dependent on actin function Notably, the differentiation of MSCs into tenocytes was associated with their elongation along the silk fibers of the scaffolds, whereas MSCs that were cultured on a silk protein-coated surface remained undifferentiated (Figure $2 \mathrm{~b}$ and $\mathrm{c}$ ). The observed correlation between MSC elongation and tenocytic differentiation suggested that cell morphology (i.e., shape) serves as a regulatory mechanism during the process of differentiation. Previous studies have revealed that the inhibition of actin polymerization disrupts contact guidance ${ }^{28}$ which may be one of the key processes responsible for the parallel elongation of MSCs along silk fibers and the subsequent differentiation of these cells. To determine the role of cytoskeletal organization in the differentiation of MSCs into tenocytes when MSCs are grown on silk, we examined the effect of cytochalasin D, which inhibits actin polymerization by capping the barbed ends of F-actin polymers, ${ }^{29}$ on silk-fiber induced MSC teno-differentiation. Differences in cell shape and cell nuclei were quantified by measuring the lengths of the cells that were growing in parallel with the fibers and were compared to the maximal widths of the cells, which were measured perpendicular to the fibers. Cells that were treated with cytochalasin $D$ did not retain an elongated shape, as demonstrated by the smaller length-to-width ratios of their cytoplasms (Figure $3 a$ and c) and nuclei (Figure $3 b$ and d) compared to untreated MSCs. Significant differences were found when quantifying the cytoplasmic $(P<0.05)$ and nuclear $(P<0.05)$ ratios (Figure $3 e)$.

\section{Actin polymerization is required for MSC elongation} and tenogenesis

To determine whether actin polymerization in elongated cells is required for tenogenic differentiation, we examined the effect of cytochalasin D on tenogenic marker gene and protein expression in MSCs that were grown on silk scaffolds for 7 days. Compared to untreated controls, cytochalasin D treatment abolished the elongated phenotype of the cells and was found to be associated
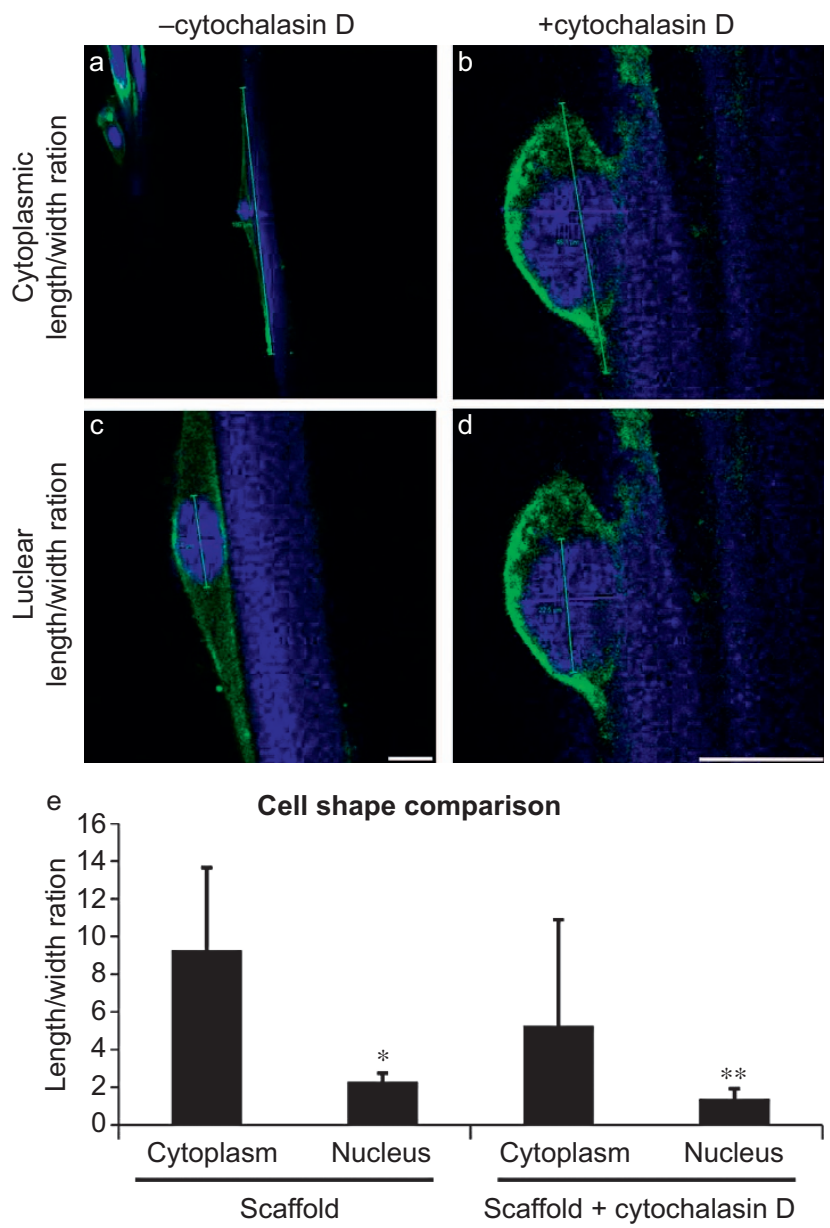

Figure 3. Cytochalasin D disrupted actin cytoskeletal arrangement in cells attached to scaffold fibers. (a) Measurements of cytoplasmic length and width of cells attached to a scaffold. (b) Measurements of nuclear length and width of cells attached to a scaffold. (c) Measurements of cytoplasmic length and width of cells attached to a scaffold treated with cytochalasin D. (d) Measurements of nuclear length and width of cells attached to a scaffold treated with cytochalasin D. (e) Graphic representation of length to width ratios demonstrating elongated cells and nuclei under normal conditions compared to rounded cells and nuclei in the presence of cytochalasin D. Data represent at least three independent experiments per assay. $* P<0.05$ compared to MSCs seeded on a scaffold without cytochalasin D. Scale bar $=25 \mu \mathrm{m}$.

with a loss of SCX and TNMD immunocytochemistry staining (Figure $4 a$ ), which was confirmed by real-time PCR and western blot (Figure 4b). To determine whether the loss of SCX and TNMD expression was due to specific effects produced by cytochalasin D on actin polymerization and cell morphology, rather than due to general toxicity related to gene expression, the expression levels of two housekeeping genes, Hypoxanthine-guanine phosphoribosyltransferase 1 (Hprtl) and phosphoglycerate kinase 1 (Pgk 1), were analyzed. The results revealed that the expression levels of both genes remained unchanged in the presence of cytochalasin D (Figure 4c), suggesting that the loss of cell elongation and tenogenic gene 
a

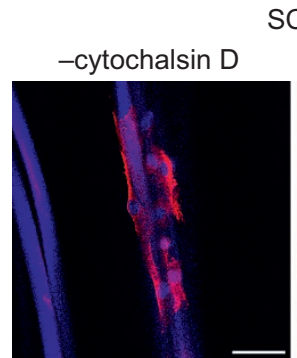

b

Real-time PCR

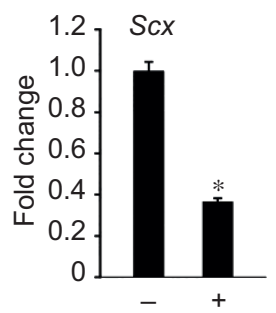

SCX

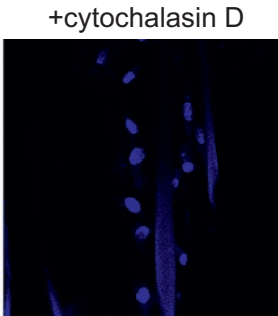

Western blot

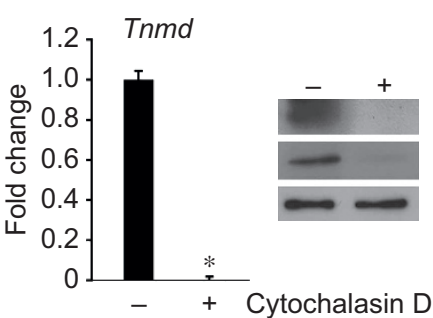

Cytochaslin D SCX

TNMD

$\beta$-actin

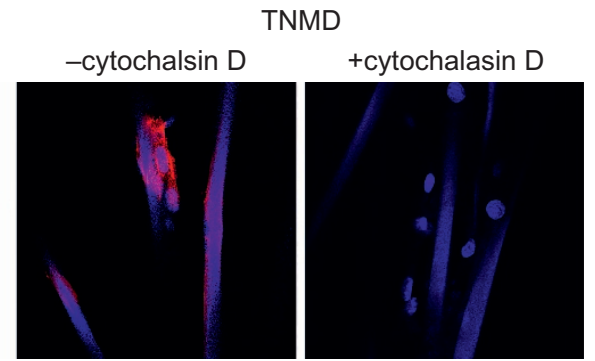

C Real-time PCR

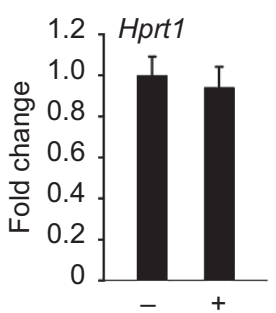

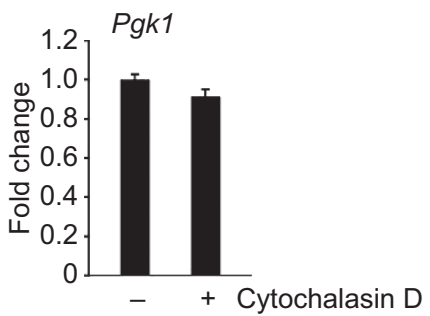

Figure 4. Cytochalasin D treatment inhibited MSC differentiation toward tenocytes. (a) Immunocytochemistry (blue $=$ DAPI, red $=$ SCX or TNMD). (b) Relative mRNA and western blot of SCX and TNMD expression in MSCs grown on a silk scaffold without or with cytochalasin D treatment. (c) Relative mRNA expression of the housekeeping genes Hrpt1 and Pgk1 in MSCs grown on a silk scaffold with and without cytochalasin D treatment. Data represent three independent experiments per assay. ${ }^{*} P<0.05$ compared to MSCs seeded on the scaffold without cytochalasin D.

expression observed previously was due to changes in actin polymerization.

Rho/Rock pathway signaling and stress fiber formation are required for tendon lineage determination

To determine the role of stress fibers in mediating silk fiberinduced MSC tenogenic differentiation, we examined the impact of cell signaling on the regulation of stress fiber formation. RhoA and ROCKII are two small GTPase proteins that have been shown to regulate the actin cytoskeleton during the formation of stress fibers. ${ }^{21}$ Accordingly, the following Rho/Rock inhibiting reagents were used to dissect the potential effects of the Rho/Rock pathway on stress fiber formation during the differentiation of MSCs (grown on silk scaffolds) into tenocytes: (1) Latrunculin A, which binds to monomeric G-actin and prevents its polymerization into F-actin and stress fibers; (2) Y-27632 dihydrochloride, which inhibits ROCK activity; and (3) blebbistatin, which selectively inhibits the ATPase function of nonmuscle myosin II.

Compared to untreated cells, cells that were exposed to the above treatment conditions lost their elongated phenotype (Figure 5a). Additionally, the expression levels of SCX mRNAs and SCX proteins were significantly decreased after treatment with Latrunculin A, Y-27632, and blebbistatin (Figure 5b), which supports that the Rho/Rock pathway plays a role in tenocyte differentiation through its regulation of stress fiber formation.

In addition to Scx, the Nfatc4 and Collal genes have been suggested to be a part of the regulatory network that is involved in the differentiation of MSCs into tenocytes. ${ }^{30}$ Therefore, we also examined the expression of Nfatc 4 and Collal following treatment with Latrunculin A, Y-27632, and blebbistatin. We found that the expression levels of both Nfatc4 and Collal were significantly decreased in response to all three of the treatments (Figure 5c). Collectively, these findings suggest that the organization of actin stress fibers is a critical determinant of MSC teno-differentiation. It is notable that the expression of the stem cell marker Nst was also markedly decreased, albeit to a lesser degree, which suggests that the Rho/ROCK pathway, actin and myosin are all important for stem cell functioning and maintenance (Figure 5c).

\section{DISCUSSION}

MSCs differentiate along specific pathways based on several cues, including the presence of various soluble factors, mechanical stimuli and matrix density ${ }^{15-16,20,31-32}$. In this study, we demonstrated that rope-like silk scaffolds provide not only a suitable environment for MSCs to grow but also a specific topography that is responsible for the induction of MSC teno-differentiation. Specifically, we demonstrated that teno-differentiation in MSCs is primarily induced by the three-dimensional structures of rope-like silk fibers and the cell-cell interactions that occur on the rope-like silk scaffold matrix and is not a result of the chemical components of the scaffold, at least in the case of silk fibers. The surface topography of silk fibers provides contact guidance, which is responsible for the scaffoldinduced, tendon-like morphological changes that were observed. 
a

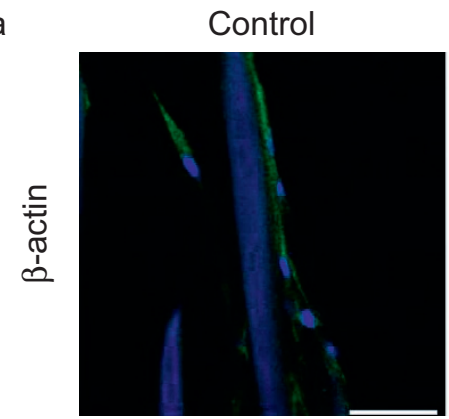

Latruncunlin A

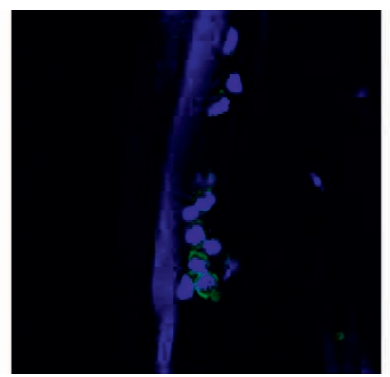

Y-27632

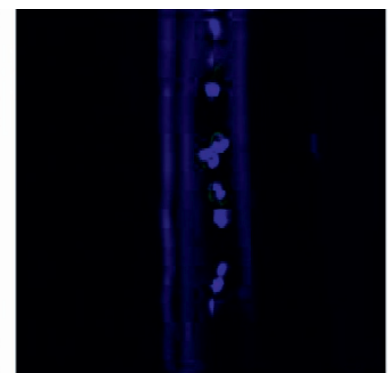

Blebbistatin

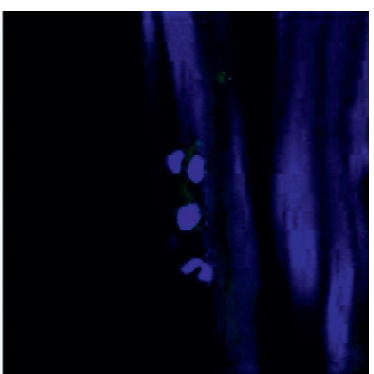

b
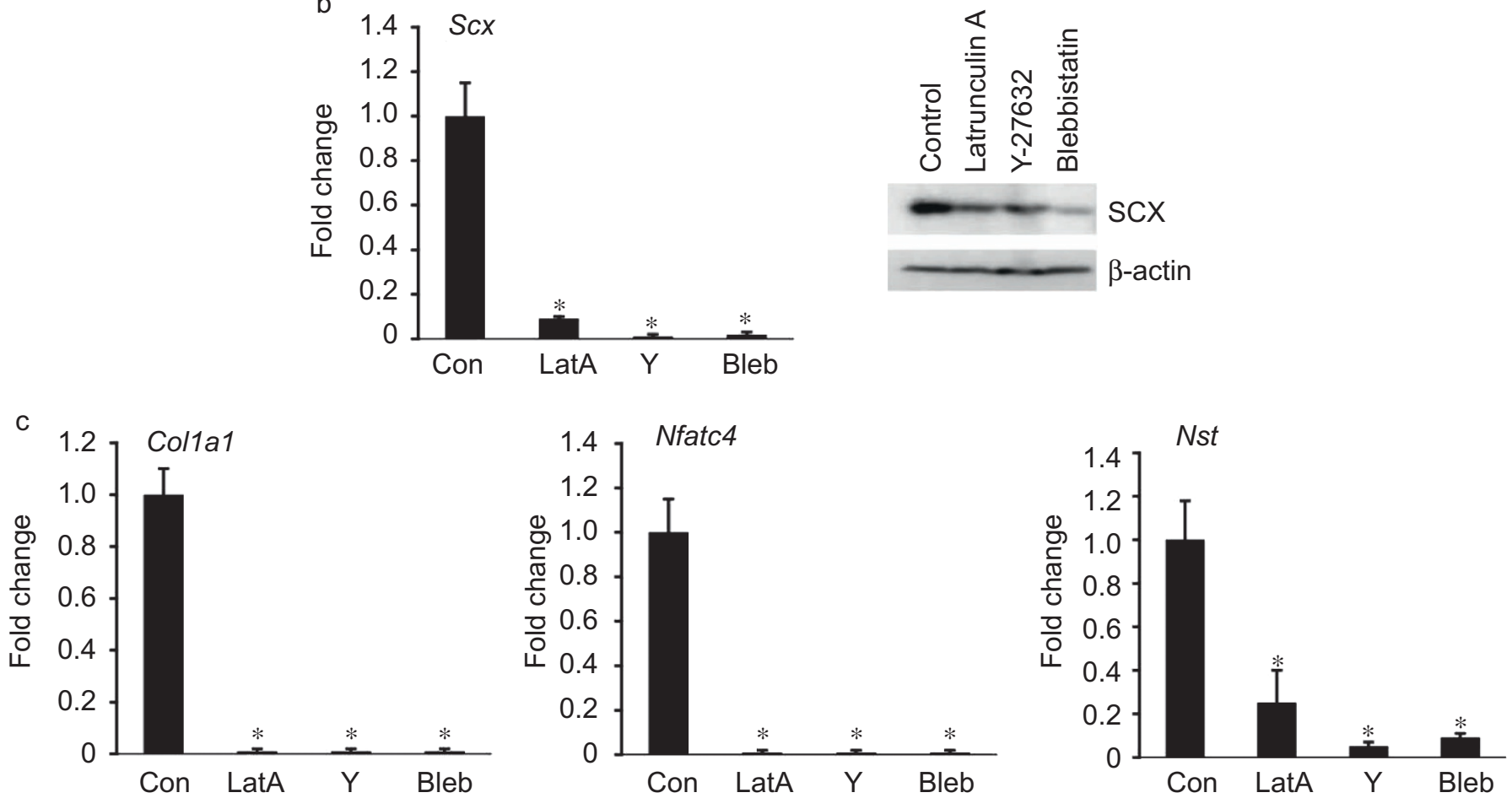

Figure 5. Rho/ Rock Pathway signaling and stress fiber formation are required for tendon lineage determination. (a) Immunocytochemistry of $\beta$-actin in MSCs grown either with no treatment or treated with Latrunculin A, Y-27632 and Blebbistatin for 7 days (blue $=\mathrm{DAPI}$, green $=\beta$-actin). ${ }^{*} P<0.05$ for all treatments. (b) mRNA expression and protein expression of Scx and mRNA expression of (c) Col1a1, Nfatc4, and Nst in MSCs grown on silk scaffolds following treatment with Latrunculin A (LatA), Y-27632 (Y) and Blebbistatin (Bleb) for 24 h. Data represent three independent experiments per assay.

The three-dimensional surface topographies of scaffolding materials may contain biophysical cues that alter cellular responses, such as gene expression, adhesion, migration, proliferation, and differentiation. ${ }^{28,33}$ This is of significance, as in our study the process of cell attachment to a silk scaffold induced morphological changes that ultimately resulted in differentiation, without the use of exogenous mechanical or biochemical stimuli. Based on our results, it was evident that the surface topography of the rope-like silk scaffold played an integral role in producing the elongated shape and differentiation of MSCs, as the cells conformed to the shape of the fibers and grew in parallel to the scaffold. Conversely, MSCs that were cultured on silk-coated plates were amorphous. These morphological changes may serve as the trigger for MSC teno-differentiation, a hypothesis that is supported by the expression of the teno-phenotypic markers scleraxis and tenomodulin. ${ }^{25-26}$ Additionally, the increased levels of nucleostemin and OCT4 expression in cells that were grown on silk-coated plates indicates that the amorphous cells that grew on flattened silk fibroin proteins remained undifferentiated. As demonstrated by our experimental results, only MSCs growing on a silk scaffold will undergo tenogenesis, which confirms that the physical properties of the three-dimensional structure of the rope-like silk scaffold are the determining factors of tenocytic differentiation, and not the chemical components of the silk fibroin. 
Because there was a strong association between MSC elongation and tenocytic differentiation, we posited that mechanisms that regulate cell morphogenesis (i.e., shape) play a required role in the differentiation process. Previous studies have revealed that inhibition of actin polymerization disrupts contact guidance, ${ }^{28}$ which is likely one of the key processes that was responsible for the parallel elongation of MSCs next to silk fibers that we observed in this study, leading to their subsequent differentiation. Therefore, cytochalasin $D$, which inhibits actin polymerization by capping the barbed ends of F-actin polymers, was used to determine how cytoskeletal organization affected the tenogenic differentiation of MSCs that were grown on silk scaffolds. Cytochalasin D treatment led the cells to adopt a rounder morphology and prevented cell elongation. The nuclei of the treated cells also became rounded. These morphological changes directly correlated with a loss of tenocyte marker gene and protein expression, as shown by real-time PCR and immunocytochemistry. Thus, disrupting the contact guidance of the fiber-like scaffold interfered with tenogenic differentiation. These data strongly suggest that interplay between the scaffold structure and the actin cytoskeletal network was a key modulator of MSC differentiation into tenocytes.

Via their attachment to integrins, actin stress fibers play an important role in cellular mechanosensing and the transduction of extracellular matrix mechanical signals. These integrins can trigger intracellular signal transduction pathways and regulate gene expression patterns that led to tenocyte differentiation. ${ }^{34}$ Mechanochemical signal pathways are likely responsible for organizing the MSC cytoskeleton in a manner that results in morphological rearrangement and differentiation into mesenchymal tissue. ${ }^{16}$ Thus, it is possible that silk fibers create a micromechanical environment that is ideal for tenocytic induction, provided that the MSCs have an intact cytoskeletal mechanism.

The Rho/ROCK pathway has been intricately linked to cell proliferation, cytoskeletal changes, and stress fiber formation. ${ }^{18,20-21}$ Previous studies have shown that the Rho/ROCK pathway has a role in regulating cell shape and differentiation into cartilage, fat, and bone, ${ }^{20}$ as well as in tenogenic differentiation occurring in response to mechanical stretch. ${ }^{22}$ Rho has been intricately linked with integrins, cell junctions, and cellular mechanics. ${ }^{35}$ Rhokinase (rock) is a downstream effector of Rho and has been directly linked to the control of myosin function. ${ }^{36}$ Inhibition of ROCK with Y-27632 has previously been reported to lead to a rounded morphology, to bypass the cell differentiation pathway toward bone, and to stimulate adipogenesis. ${ }^{20}$ In our study, when using Y-27632, we noted a marked decrease in elongated morphology as well as a decrease in tendon marker expression. To further elucidate the role of the Rho/ROCK pathway in silk fiber-induced tenogenesis, we tested whether intact stress fiber formation was a pre-requisite for achieving the cell shape or focal adhesions necessary for cell differentiation. To accomplish this, we inhibited non-muscle myosin II, which is a downstream player in the Rho/ROCK pathway and an important component of stress fiber formation. ${ }^{37}$ Stress fiber formation is a crucial element of cell cytoskeletal regulatory mechanisms and would therefore be expected to affect tensiondependent differentiation. Using blebbistatin to inhibit non-muscle myosin II function, we were able to show that stress fiber-related tension is indeed necessary for creating morphological changes in cells.

\section{CONCLUSION}

In this study, we demonstrated that MSC differentiation toward a tenogenic lineage could be induced using rope-like silk scaffolds alone. The process of induction was dependent on the topography of the silk fibers and could not be completed without the tension and cell shape regulatory mechanisms of the Rho/ROCK pathway. This study indicates that topographical factors that induce cell morphological changes are required for the differential commitment of MSCs. These findings may lead to novel strategies for scaffold development, tendon tissue engineering, and targeting microenvironments for stem cell-based tendon regeneration and repair.

\section{Competing interests}

The authors declare no conflict of interest.

\section{Acknowledgements}

This work was funded in part by NIH R01AG039561 (HBS).

\section{References}

1 Hawkes R, O'Connor P, Campbell D. The prevalence, variety and impact of wrist problems in elite professional golfers on the European Tour. $\mathrm{Br} J$ Sports Med 2013; 47: 1075-1079.

2 Swenson DM, Collins CL, Best TM, Flanigan DC, Fields SK, Comstock RD. Epidemiology of knee injuries among U.S. high school athletes, 2005/ 2006-2010/2011. Med Sci Sports Exerc 2013; 45: 462-469.

3 McCarthy MM, Voos JE, Nguyen JT, Callahan L, Hannafin JA. Injury profile in elite female basketball athletes at the Women's National Basketball Association combine. Am J Sports Med 2013; 41: 645-651.

4 Sereysky JB, Flatow EL, Andarawis-Puri N. Musculoskeletal regeneration and its implications for the treatment of tendinopathy. Int J Exp Pathol 2013; 94: 293-303.

5 Liu CF, Aschbacher-Smith L, Barthelery NJ, Dyment N, Butler D, Wylie C. What we should know before using tissue engineering techniques to repair injured tendons: a developmental biology perspective. Tissue Eng Part B Rev 2011; 17: 165-176.

6 Yang G, Rothrauff BB, Tuan RS. Tendon and ligament regeneration and repair: Clinical relevance and developmental paradigm. Birth Defects Res C Embryo Today 2013; 99: 203-222. 
7 Yin Z, Chen X, Chen JL, Ouyang HW. Stem cells for tendon tissue engineering and regeneration. Expert Opin Biol Ther 2010; 10: 689-700.

8 Cheng MT, Yang HW, Chen TH, Lee OK. Isolation and characterization of multipotent stem cells from human cruciate ligaments. Cell Prolif 2009; 42 : 448-460.

9 Bi Y, Ehirchiou D, Kilts TM et al. Identification of tendon stem/progenitor cells and the role of the extracellular matrix in their niche. Nat Med 2007; 13: $1219-1227$

10 Lee JY, Zhou Z, Taub PJ et al. BMP-12 treatment of adult mesenchymal stem cells in vitro augments tendon-like tissue formation and defect repair in vivo. PLoS One 2011; 6: e17531.

11 Altman GH, Diaz F, Jakuba C et al. Silk-based biomaterials. Biomaterials 2003; 24: 401-416.

12 Teh TK, Toh SL, Goh JC. Aligned fibrous scaffolds for enhanced mechanoresponse and tenogenesis of mesenchymal stem cells. Tissue Eng Part A 2013; 19: 1360-1372.

13 Teh TK, Toh SL, Goh JC. Aligned hybrid silk scaffold for enhanced differentiation of mesenchymal stem cells into ligament fibroblasts. Tissue Eng Part C Methods 2011; 17: 687-703.

14 Fan H, Liu H, Toh SL, Goh JC. Anterior cruciate ligament regeneration using mesenchymal stem cells and silk scaffold in large animal model. Biomaterials 2009; 30: 4967-4977.

15 Engler AJ, Sen S, Sweeney HL, Discher DE. Matrix elasticity directs stem cell lineage specification. Cell 2006; 126: 677-689.

16 McBeath R, Pirone DM, Nelson CM, Bhadriraju K, Chen CS. Cell shape, cytoskeletal tension, and RhoA regulate stem cell lineage commitment. Dev Cell 2004; 6: 483-495.

17 Sahai E, Marshall CJ. ROCK and Dia have opposing effects on adherens junctions downstream of Rho. Nat Cell Biol 2002; 4: 408-415.

18 Maekawa M, Ishizaki T, Boku S et al. Signaling from Rho to the actin cytoskeleton through protein kinases ROCK and LIM-kinase. Science 1999; 285: 895-898.

19 Leung T, Chen XQ, Manser E, Lim L. The p160 RhoA-binding kinase ROK alpha is a member of a kinase family and is involved in the reorganization of the cytoskeleton. Mol Cell Biol 1996; 16: 5313-5327.

20 Arnsdorf EJ, Tummala P, Kwon RY, Jacobs CR. Mechanically induced osteogenic differentiation--the role of RhoA, ROCKII and cytoskeletal dynamics. J Cell Sci 2009; 122: 546-553.

21 Pellegrin S, Mellor H. Actin stress fibres. J Cell Sci 2007; 120: 3491-3499.

22 Xu B, Song G, Ju Y, Li X, Song Y, Watanabe S. RhoA/ROCK, cytoskeletal dynamics, and focal adhesion kinase are required for mechanical stretchinduced tenogenic differentiation of human mesenchymal stem cells. J Cell Physiol 2012; 227: 2722-2729.

23 Shao Z, Vollrath F. Surprising strength of silkworm silk. Nature 2002; 418: 741 .
24 Jiang Y, Chen H, Zhou W, Hua J, Zheng Q, Xiong W. [Research on preparation of silk fibroin and its biocompatibility with rat bone marrow mesenchymal stem cells]. Sheng Wu Yi Xue Gong Cheng Xue Za Zhi 2006; 23: 560-564.

25 Schweitzer R, Chyung JH, Murtaugh LC et al. Analysis of the tendon cell fate using Scleraxis, a specific marker for tendons and ligaments. Development 2001; 128: 3855-3866.

26 Shukunami C, Takimoto A, Oro M, Hiraki Y. Scleraxis positively regulates the expression of tenomodulin, a differentiation marker of tenocytes. Dev Biol 2006; 298: 234-247.

27 Tsai RY, McKay RD. A nucleolar mechanism controlling cell proliferation in stem cells and cancer cells. Genes Dev 2002; 16: 2991-3003.

28 Gerecht S, Bettinger CJ, Zhang Z, Borenstein JT, Vunjak-Novakovic G, Langer $\mathrm{R}$. The effect of actin disrupting agents on contact guidance of human embryonic stem cells. Biomaterials 2007; 28: 4068-4077.

29 Carlier MF, Criquet P, Pantaloni D, Korn ED. Interaction of cytochalasin D with actin filaments in the presence of ADP and ATP. J Biol Chem 1986; 261: 2041-2050

30 Léjard V, Brideau G, Blais F et al. Scleraxis and NFATc regulate the expression of the pro-alpha1(I) collagen gene in tendon fibroblasts. $J$ Biol Chem 2007; 282: 17665-17675.

31 Meinel L, Karageorgiou V, Hofmann S et al. Engineering bone-like tissue in vitro using human bone marrow stem cells and silk scaffolds. J Biomed Mater Res A 2004; 71: 25-34.

32 Wang SP, Guo TQ, Guo XY, Huang JT, Lu CD. Structural analysis of fibroin heavy chain signal peptide of silkworm Bombyx mori. Acta Biochim Biophys Sin (Shanghai) 2006; 38: 507-513.

33 Kim DH, Provenzano PP, Smith CL, Levchenko A. Matrix nanotopography as a regulator of cell function. J Cell Biol 2012; 197: 351-360.

34 Ghosh K, Ingber DE. Micromechanical control of cell and tissue development: implications for tissue engineering. Adv Drug Deliv Rev 2007; 59: 1306-1318

35 Chiquet M, Gelman L, Lutz R, Maier S. From mechanotransduction to extracellular matrix gene expression in fibroblasts. Biochim Biophys Acta 2009; 1793: 911-920.

36 Kimura K, Ito M, Amano M et al. Regulation of myosin phosphatase by Rho and Rho-associated kinase (Rho-kinase). Science 1996; 273: 245-248.

37 Tojkander S, Gateva G, Lappalainen P. Actin stress fibers - assembly, dynamics and biological roles. J Cell Sci 2012; 125: 1855-1864.

(1) This work is licensed under a Creative Commons Attribution Br ${ }^{2}$ No NonCommercial-NoDerivs 4.0 Unported License. The images or other third party material in this article are included in the article's Creative Commons license, unless indicated otherwise in the credit line; if the material is not included under the Creative Commons license, users will need to obtain permission from the license holder to reproduce the material. To view a copy of this license, visit http://creativecommons.org/licenses/by-nc-nd/4.0/ 